\title{
BMJ Open Understanding autism spectrum disorder and social functioning in children with neurofibromatosis type 1: protocol for a cross-sectional multimodal study
}

\author{
Kristina M Haebich, ${ }^{1,2}$ Natalie A Pride, ${ }^{3,4}$ Karin S Walsh, ${ }^{5,6}$ Anita Chisholm, ${ }^{1,2}$ \\ Melissa Rouel, ${ }^{3}$ Alice Maier, ${ }^{1}$ Vicki Anderson, ${ }^{1,2}$ Belinda Barton, ${ }^{3,4,7}$ Tim Silk, ${ }^{8}$ \\ Mayuresh Korgaonkar, ${ }^{9}$ Marc Seal, ${ }^{10}$ Francesca Lami, ${ }^{1}$ Jennifer Lorenzo, ${ }^{3}$ \\ Katrina Williams, ${ }^{11}$ Gabriel Dabscheck, ${ }^{12}$ Caroline D Rae, ${ }^{13}$ Michael Kean, ${ }^{14}$ \\ Kathryn N North, ${ }^{2,15}$ Jonathan M Payne ${ }^{1,2}$
}

To cite: Haebich KM, Pride NA, Walsh KS, et al. Understanding autism spectrum disorder and social functioning in children with neurofibromatosis type 1 : protocol for a cross-sectional multimodal study. BMJ Open 2019;9:e030601. doi:10.1136/ bmjopen-2019-030601

- Prepublication history for this paper is available online. To view these files, please visit the journal online (http://dx.doi org/10.1136/bmjopen-2019030601).

Received 22 March 2019 Revised 02 August 2019 Accepted 02 September 2019

Check for updates

(c) Author(s) (or their employer(s)) 2019. Re-use permitted under CC BY-NC. No commercial re-use. See rights and permissions. Published by BMJ.

For numbered affiliations see end of article.

Correspondence to Dr Jonathan M Payne; jonathan.payne@mcri.edu.au

\section{ABSTRACT}

Introduction Children with the single-gene disorder neurofibromatosis type 1 (NF1) appear to be at an increased risk for autism spectrum disorder (ASD) and exhibit a unique social-cognitive phenotype compared with children with idiopathic ASD. A complete framework is required to better understand autism in NF1, from neurobiological levels through to behavioural and functional outcomes. The primary aims of this study are to establish the frequency of ASD in children with NF1, examine the social cognitive phenotype, investigate the neuropsychological processes contributing to ASD symptoms and poor social functioning in children with $\mathrm{NF} 1$, and to investigate novel structural and functional neurobiological markers of ASD and social dysfunction in NF1. The secondary aim of this study is to compare the neuropsychological and neurobiological features of ASD in children with NF1 to a matched group of patients with idiopathic ASD.

Methods and analysis This is an international, multisite, prospective, cross-sectional cohort study of children with NF1, idiopathic ASD and typically developing (TD) controls. Participants will be 200 children with NF1 (3-15 years of age), 70 TD participants (3-15 years) and 35 children with idiopathic ASD (7-15 years). Idiopathic ASD and NF1 cases will be matched on age, sex and intelligence. All participants will complete cognitive testing and parents will rate their child's behaviour on standardised questionnaires. Neuroimaging will be completed by a subset of participants aged 7 years and older. Children with NF1 that screen at risk for ASD on the parent-rated Social Responsiveness Scale 2nd Edition will be invited back to complete the Autism Diagnostic Observation Scale 2nd Edition and Autism Diagnostic Interview-Revised to determine whether they fulfil ASD diagnostic criteria.

Ethics and dissemination This study has hospital ethics approval and the results will be disseminated through peer-reviewed publications and international conferences.

\section{Strengths and limitations of this study}

- Gold standard assessment of autism spectrum disorder (ASD) using the clinician rated Autism Diagnostic Interview-Revised and the Autism Diagnostic Observation Scale 2nd Edition to determine the frequency of ASD in children with neurofibromatosis type 1 (NF1)

- An explanatory framework for understanding ASD in NF1, incorporating markers of brain development and neurocognitive performance with behavioural symptoms and functional outcomes.

- This study will help guide the development and implementation of developmentally appropriate and effective interventions for children with NF1 and ASD or those with impairing social deficits.

- The relatively small number of idiopathic ASD participants $(n=35)$ may not capture the full extent of clinical heterogeneity in the disorder, limiting the ability to compare and contrast the ASD phenotype in NF1 to the idiopathic disorder.

\section{INTRODUCTION}

Autism spectrum disorder (ASD) is a neurodevelopmental disorder characterised by impairments in social communication, and in restricted interests and repetitive behaviours that result in pervasive social challenges and reduced quality of life. In the general population, ASD is a heterogeneous disorder resulting from complex gene-environment interactions. ${ }^{1}$ Genetic influences are a particularly strong component of ASD aetiology, evidenced by heritability estimates of $83 \%-90 \%$ in recent meta-analyses of twin studies, and greater ASD concordance in the context of increased genetic 
relatedness. ${ }^{23}$ Despite clear genetic underpinnings, there is striking genetic heterogeneity with over 1000 candidate genes reported to be related to ASD. ${ }^{45}$ Such aetiological complexity has proven a significant challenge in understanding the molecular and neurobiological mechanisms underlying this disorder.

In a subset of children, ASD co-occurs with a clinically defined syndrome, many of which arise from a known single gene mutation. ${ }^{67}$ The significant reductions in genetic heterogeneity in these 'syndromic' forms of ASD enable the molecular and neurobiological pathways critical to ASD to be better understood, making it possible to identify distinct neurodevelopmental subtypes of ASD within these monogenetic syndromes. One such syndrome is neurofibromatosis type 1 (NF1), an autosomal dominant disorder caused by loss-of-function mutations within the $N F 1$ gene. With a birth incidence of 1 in 2700, NF1 is one of the most common monogenic conditions to affect the central nervous system. ${ }^{8}$ Although general intellectual functioning typically falls within the lower limit of the normal range (eg, low 90s), specific cognitive deficits are the greatest cause of morbidity in children with NF1, with up to $80 \%$ experiencing deficits in at least one cognitive domain. ${ }^{9}$ Attention, executive function, visuoperception and language are most often affected. ${ }^{9}{ }^{10}$ There is also increasing evidence to indicate that social cognitive deficits are another core feature of the NF1 phenotype. ${ }^{11}$ Deficits in face and emotion recognition have been reported in school-aged children with NF1, including difficulties detecting negative emotions (eg, anger) and discerning emotions from more ambiguous representations. ${ }^{12-14}$ Higher-level social cognitive deficits are also reported, including difficulties attributing mental representations and intent to others (eg, theory of mind). ${ }^{15}$

Children with NF1 are at an increased risk for ASD. At the group level, recent meta-analysis has demonstrated large effect sizes for ASD symptomatology across eight studies of individuals with NF1 (Hedges' $\mathrm{g}=0.9$ ).${ }^{11}$ Results from a large international pooled dataset of 531 individuals with NF1 indicated that $39 \%$ of patients demonstrate at least subthreshold ASD symptoms on the Social Responsiveness Scale 2nd Edition (SRS-2), with 13\% scoring in the most severe range. ${ }^{16}$ To date, only a handful of studies have employed clinic-based assessments to establish the prevalence of ASD in NF1, ${ }^{17-19}$ returning estimates of $11 \%-25 \%$ in school-aged children; significantly higher than rates observed in the general population $(\sim 1 \%) .{ }^{20}$

There are a number of phenotypic similarities between children with NF1 and idiopathic ASD. Both experience social interaction difficulties, ${ }^{21}$ hyperactivity ${ }^{22}$ and anxiety ${ }^{23}$ In line with current conceptual frameworks, ${ }^{24}$ both groups also demonstrate executive deficits (eg, cognitive inflexibility, planning) and reduced social cognitive abilities, which may contribute to the ASD symptoms and social difficulties. ${ }^{14} 1525$ However, between group differences are also observed. ${ }^{1626}$ For example, children with NF1 appear to demonstrate fewer repetitive behaviours and better language skills than children with idiopathic ASD. ${ }^{17}$ Further, idiopathic ASD is often associated with intellectual impairment, whereas children with NF1-related ASD typically demonstrate intelligence estimates within the average range. ${ }^{10}{ }^{19}$ The strong male:female bias in idiopathic ASD (4:1) ${ }^{27}$ also appears to be attenuated in NF1, with estimates at $1.6-2.6: 1 .{ }^{16}{ }^{28}$ On average, the age children with NF1 receive a diagnosis of ASD is 10.65 years, ${ }^{17}$ which is significantly later than the idiopathic condition, which is around 4 years. ${ }^{29}$ While these data suggest that autism symptoms are typically not identified by parents or health professionals of children with NF1 until 8-10 years of age, ${ }^{1630}$ the possible 'masking role' of neurodevelopmental comorbidities, such as attention deficit/hyperactivity disorder, and the complex medical issues experienced by children with NF1 is unclear.

Despite the recent advances into understanding ASD in NF1 over the last few years, many critical questions remain. Indeed, a complete explanatory framework for understanding ASD in a single-gene model such as NF1 requires multiple levels of analysis in order to delineate the effect of NF1 mutation on gene expression, cell signalling, brain development and function, and neurocognitive performance as well as behavioural symptoms and functional outcomes. ${ }^{31}$ In NF1, loss of the gene product neurofibromin causes disinhibition of the rat sarcoma viral oncogene homolog-mitogen-activated protein kinase (RAS-MAPK) signalling cascade, resulting in abnormal brain development. ${ }^{32} 33$ Aberrant cellular signalling further triggers abnormal GABAergic neurotransmission, impaired long-term potentiation and a loss of synaptic plasticity. ${ }^{34} 35$ Presently, the neurobiological mechanisms underlying NF1-related ASD symptoms and functional impairments remain unclear. This study will explore the neurobiological, cognitive, behavioural and functional phenotype in children with NF1 and how each of these levels of analysis contribute to the ASD phenotype. Further, the majority of research to date has estimated NF1-related ASD symptoms based on parent-reported measures. To obtain a deeper understanding of the ASD phenotype in NF1, we will use the current gold-standard combination of the clinician-rated Autism Diagnostic Interview-Revised (ADI-R) with the Autism Diagnostic Observation Scale 2nd Edition (ADOS-2). These data will enable us to provide greater certainty in the ASD prevalence rate in NF1, and to identify potentially important similarities and differences between the symptom profiles of NF1-related ASD and idiopathic ASD.

The primary aims of this study are to (1) comprehensively phenotype ASD-like behaviours and establish the frequency of ASD in children with NF1, (2) examine the social cognitive phenotype of children with NF1, (3) identify the neuropsychological processes contributing to ASD symptoms and poor social functioning in children with NF1 and, (4) investigate novel structural and functional neurobiological markers of ASD and social dysfunction in children with NF1. The secondary aim of this study is to compare cognitive and symptom profiles as well as 


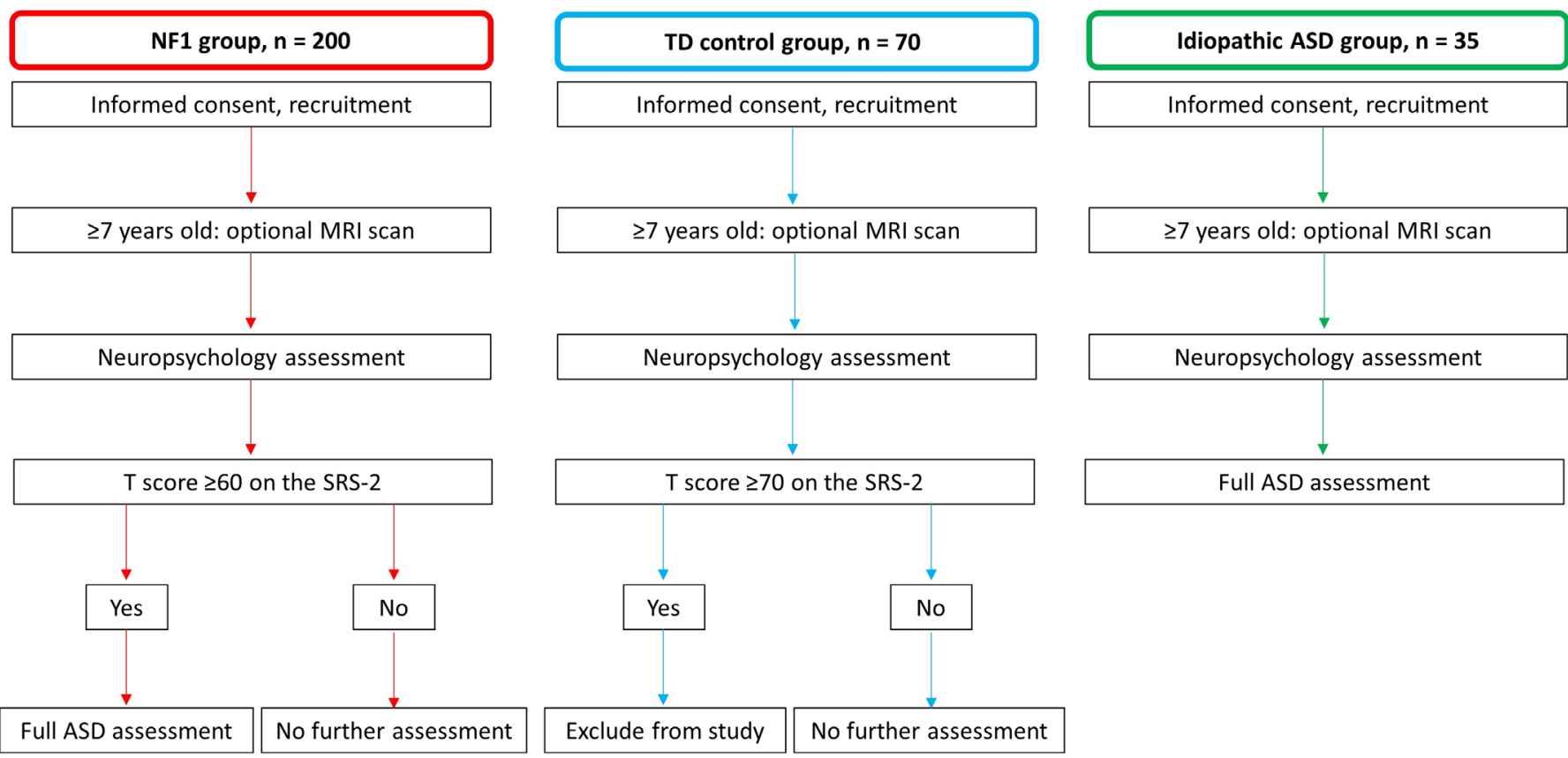

Figure 1 Diagram of study design for all groups. ASD, autism spectrum disorder; NF1, neurofibromatosis type 1; TD, typically developing.

neurobiological markers of ASD in children with NF1 to a matched group of patients with idiopathic ASD.

\section{METHODS AND ANALYSIS Study design}

This is an international, multisite, prospective, cross-sectional cohort study of children with NF1, idiopathic ASD and typically developing (TD) controls. Participants will complete a detailed assessment of their cognitive abilities, behaviour and adaptive functioning. As part of this assessment, participants will be screened for ASD symptoms. All idiopathic ASD participants, as well as NF1 participants that screen at risk for ASD (see Procedure section for further details), will complete a comprehensive assessment that will be used to guide the formulation of research and clinical ASD diagnoses (see ASD assessment measures section for further details). Children aged $\geq 7$ years will also be invited to undergo multimodal MRI (Australian sites only). An overview of the study design for each group is provided in figure 1 .

\section{Participants and recruitment}

Three groups will be recruited; children with NF1 ( $n=200$; $3-15$ years of age), children with idiopathic ASD $(n=35$; 7-15 years of age) and TD controls from the general population $(n=70 ; 3-15$ years of age). Prospective participants with NF1 will be recruited from three international genetic centres; (1) the Neurofibromatosis Clinic at the Royal Children's Hospital/Murdoch Children's Research Institute (MCRI), Melbourne, Australia; (2) the Neurogenetics Clinic at The Children's Hospital at Westmead (CHW), Sydney, Australia; and (3) the Gilbert Neurofibromatosis Institute at the Children's National Health
System, Washington DC, USA. Children are referred to these clinics by general practitioners and medical specialists for evaluation, diagnosis and management of NF1. All three clinics are specialist centres for the multidisciplinary care of individuals with NF1, are well resourced for the collection of cognitive and behavioural research data, and service clinical populations thought to be representative of the wider NF1 community. NF1 participants will be diagnosed with NF1 by an expert neurologist or clinical geneticist based on criteria specified by the National Institutes of Health Conference Statement. ${ }^{36}$ The study coordinator at each site will recruit NF1 participants. The coordinator will approach families attending the clinic and inquire about interest in the study. To minimise selection bias, the study coordinator will sequentially approach families with a child in the defined age range.

Participants with idiopathic ASD will be recruited at the MCRI site from local clinical services and from families known to existing studies who have previously indicated a willingness to be contacted for future research. All idiopathic ASD participants will have received an ASD diagnosis by a clinician prior to enrolment and have no known genetic disorders associated with ASD. They will be matched to NF1 participants with a comorbid diagnosis of ASD on age, sex and intelligence. Given that $\sim 94 \%$ of children with NF1 present with an intelligence quotient (IQ) $>70,{ }^{37}$ the majority of recruited children with idiopathic ASD will not have evidence of an intellectual disability based on records of intellectual function to provide a suitable match.

Control participants will be recruited via several methods. First, we will invite individuals that have participated as TD controls in previous research studies and 
provided consent to be recontacted for future studies. Contact will initially take the form of a mail out of the Parent Information and Consent Form and a cover letter inviting them to contact the site study coordinator if they would like to participate. A follow-up phone call will be made 2 weeks later to ascertain interest in participating. Second, approved advertisements will be placed on hospital noticeboards inviting interested participants to contact the site investigator for more information about the study.

\section{Exclusion criteria for all participants are}

1. Participant and at least one parent/guardian not fluent in English.

2. Significant sensory impairment that limits the validity of psychometric testing.

3. Symptomatic intracranial pathology that may impact cognitive and behavioural function, such as an acquired brain injury, hydrocephalus or progressive intracranial tumours (children with asymptomatic lesions such as optic gliomas will be eligible).

Additional exclusion criteria that applies to TD control participants only

1. Positive history of a neurological, genetic or psychological disorder.

2. Developmental delay/intellectual disability.

The first participant was recruited to this study in June 2016 and we anticipate the end date for enrolment to be December 2020.

\section{Procedure}

Once informed consent has been obtained, parents/ caregivers will complete a semistructured interview with a member of the study team in order to determine eligibility, confirm demographic details (date of birth, language spoken at home, school grade), provide socioeconomic information (primary caregiver's highest level of education, occupation and employment status), and a detailed developmental/medical history will also be taken. Eligible children will then undergo cognitive assessment individually with a site psychologist in a quiet room. Study personnel will follow a test administration protocol to minimise between-site variation. Children exhibiting fatigue during the assessment will complete the testing over multiple days.

Parents/caregivers will complete detailed questionnaires covering a range of behavioural and functional outcomes, including the SRS-2. NF1 participants that screen at risk for ASD on the basis of their SRS-2 results (total symptom T-score $\geq 60$ ) will be invited to complete the diagnostic ASD assessment. Idiopathic ASD participants will also complete the ASD assessment so that a comprehensive understanding of their behavioural profile can be obtained. These will be audiotaped and videotaped respectively, so that independent blinded double interrater coding can be completed in $25 \%$ of the sample.
Children aged $\geq 7$ years who are able to complete a brain MRI safely will be offered the option to undergo neuroimaging. Neuroimaging will only take place at Australian sites.

\section{Measures}

Cognitive and behavioural measures

Cognitive domains selected for assessment are based on a biopsychosocial model for social functioning that integrates abilities thought to underlie the development and expression of social behaviour, including attention/ executive function, communication and social cognitive skills. ${ }^{24}$ To ensure appropriate age-normed tests are administered, participants will be grouped into a younger cohort of children aged 3-5 years, and a school-aged cohort of children aged 6-15 years. Child-direct assessment measures for each cohort are presented in table 1 . Parent-reported measures are outlined in table 2.

\section{ASD assessment measures}

The ADI- $\mathrm{R}^{38}$ is a semistructured, standardised diagnostic interview designed to assess core aspects of ASD. The ADI-R is administered to a parent/caregiver by a trained clinician and consists of 95 items covering current and previous behaviour across the areas of family background, developmental history, language, communication, social development, interests and general behaviour. Items are coded according to the examiner's judgement of the presence/absence or the extent of a given behaviour using a scale ranging from 0 (behaviour not present) to 3 (definite abnormality, marked in severity). An algorithm is used to code summary scores for the three domains required for diagnosis: social interaction, communication and restricted and repetitive behaviours. Diagnostic criteria for ASD are met when all three domain scores exceed the following cut-offs: social interaction domain $\geq 10$; communication $\geq 8$; and restricted interests and repetitive behaviours $\geq 3$. The ADI-R is effective in differentiating groups of children with and without ASD, and discriminating autism symptomology. ${ }^{38}$

The ADOS-2 ${ }^{39}$ is a semi-structured, standardised child-direct observational assessment designed to assess reciprocal social interaction and communication, play and use of imagination. It consists of four modules, of which one will be administered depending on the participant's developmental age and expressive language ability: (1) preverbal/have single word language; (2) phrase speech abilities; (3) verbally fluent children/adolescents; and (4) verbally fluent adolescents/adults. Each module takes $\sim 30$ min to complete by a trained examiner. For each module, individual items are scored on a three point scale ranging from 0 (no evident abnormality) to 3 (marked abnormality). Module observations are scored according to the ADOS-2 diagnostic algorithm under two domains; social affect and restricted and repetitive behaviours. A combined domain total of $\geq 7$ is classified as meeting diagnostic criteria for ASD, consistent with 
Table 1 Cognitive assessment measures

\begin{tabular}{|c|c|c|c|}
\hline Domain & Measure & Description & Cohort \\
\hline \multicolumn{4}{|l|}{ Intelligence } \\
\hline \multirow[t]{2}{*}{ General intelligence } & WPPSI-IV 63 & 10 subtests providing 5 indices and full scale IQ & Y \\
\hline & WISC-V ${ }^{64}$ & 10 subtests providing 5 indices and full scale IQ & $\mathrm{S}$ \\
\hline
\end{tabular}

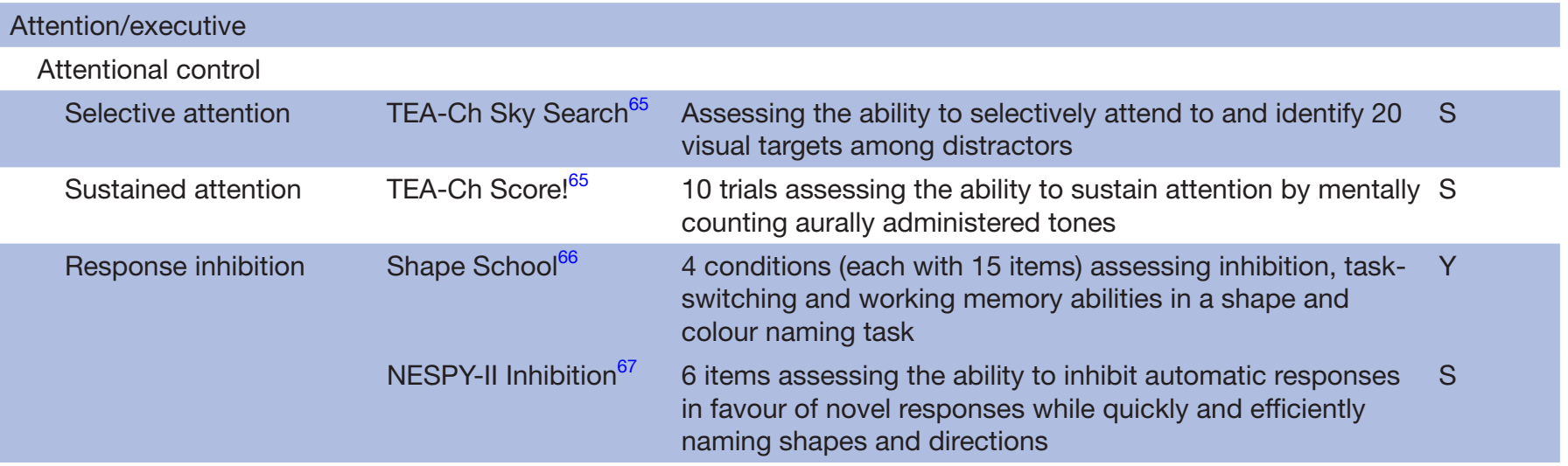

Cognitive flexibility/goal setting

\begin{tabular}{|c|c|c|c|}
\hline \multirow[t]{2}{*}{ Working memory } & From WPPSI IV ${ }^{63}$ & 2 core subtests assessing visual working memory & Y \\
\hline & From WISC-V ${ }^{68}$ & $\begin{array}{l}2 \text { core subtests assessing visual working memory and verbal } \\
\text { working memory }\end{array}$ & $s$ \\
\hline Planning & Tower of Hanoi ${ }^{69}$ & $\begin{array}{l}6 \text { items assessing set shifting, response inhibition, working } \\
\text { memory and the ability to hold a set of rules in mind in order } \\
\text { to reach an end-state goal }\end{array}$ & $Y$ \\
\hline Attentional shifting & $\begin{array}{l}\text { TEA-Ch Creature } \\
\text { Counting }\end{array}$ & $\begin{array}{l}7 \text { trials assessing the ability to accurately switch and redirect } \\
\text { attention to count up/down }\end{array}$ & $s$ \\
\hline
\end{tabular}

Social cognition

Faces/emotion perception

\begin{tabular}{|c|c|c|c|}
\hline Emotion perception & $\begin{array}{l}\text { NEPSY-II Affect } \\
\text { Recognition }\end{array}$ & $\begin{array}{l}36 \text { items assessing the ability to match facial expressions } \\
\text { from photographs of children's faces }\end{array}$ & $\mathrm{Y}, \mathrm{S}$ \\
\hline Face perception & $\begin{array}{l}\text { Benton Facial } \\
\text { Recognition Test }\end{array}$ & $\begin{array}{l}13 \text { items assessing the ability to recognise a target face from } \\
\text { a selection of distractors }\end{array}$ & S \\
\hline \multicolumn{4}{|c|}{ Mentalising/theory of mind } \\
\hline & NEPSY-II ToM ${ }^{67}$ & $\begin{array}{l}21 \text { items assessing the ability to comprehend the } \\
\text { perspectives, intentions and beliefs of another person }\end{array}$ & Y, S \\
\hline & Faux Pas Task ${ }^{73}$ & $\begin{array}{l}20 \text { short stories assessing the ability to identify a social faux } \\
\text { pas }\end{array}$ & $\mathrm{S}$ \\
\hline & Strange Stories ${ }^{74}$ & $\begin{array}{l}14 \text { short stories assessing the ability to attribute mental } \\
\text { states (eg, desires, beliefs or intentions) or perceive what } \\
\text { a character knows, as well as four control comprehension } \\
\text { stories }\end{array}$ & $\mathrm{S}$ \\
\hline
\end{tabular}

Communication

$\begin{array}{lll}\text { Expressive language } & \text { CELF-Preschool-2 } 2^{75} & \begin{array}{l}2 \text { subtests assessing knowledge of grammatical rules in a } \\ \text { sentence completion task and the ability to name objects, } \\ \text { people and activities }\end{array} \\ \begin{array}{ll}\text { CELF-4 Formulated } \\ \text { Sentences }^{76}\end{array} & \begin{array}{l}28 \text { items assessing the ability to formulate semantically and } \\ \text { grammatically correct spoken sentences using given words } \\ \text { (eg, car), based on an illustration }\end{array}\end{array}$


Table 1 Continued

\begin{tabular}{clll}
\hline Domain & Measure & Description & Cohort \\
\hline Receptive language & CELF-Preschool-2 & $\begin{array}{l}\text { 22 items assessing the ability to interpret spoken sentences } \\
\text { of increasing length and complexity }\end{array}$ & $\mathrm{Y}$ \\
& $\begin{array}{l}\text { NEPSY-II } \\
\text { Comprehension of }^{\text {Instructions }}{ }^{67}\end{array}$ & $\begin{array}{l}33 \text { items assessing the ability to comprehend and follow } \\
\text { multistep instructions of increasing complexity }\end{array}$ & $\mathrm{S}$ \\
& & & \\
\hline
\end{tabular}

CELF, clinical evaluation of language fundamentals; NEPSY, a developmental neuropsychological assessment; S, school age cohort; TEA-Ch, test of everyday attention for children; ToM, theory of mind; WIAT-II, Wechsler individual achievement test, 2nd edition; WISC-V, Wechsler intelligence scale for children, 5th edition; WPPSI IV, Wechsler preschool and primary scale of intelligence, 4th edition; Y, young cohort.

\begin{tabular}{|c|c|c|c|}
\hline Domain & Measure & Description & Cohort \\
\hline $\begin{array}{l}\text { ASD } \\
\text { symptomatology }\end{array}$ & SRS- $2^{77}$ & $\begin{array}{l}65 \text { items assessing the presence and severity of ASD symptoms } \\
\text { including social awareness, social cognition, social communication, } \\
\text { social motivation and restricted interests and repetitive behaviour }\end{array}$ & Y, S \\
\hline \multirow[t]{2}{*}{$\begin{array}{l}\text { ADHD } \\
\text { symptomatology }\end{array}$} & $\mathrm{CADS}^{78}$ & $\begin{array}{l}26 \text { items assessing ADHD symptoms of impulsivity/hyperactivity and } \\
\text { inattention }\end{array}$ & Y \\
\hline & Conners $3^{79}$ & $\begin{array}{l}110 \text { items assessing ADHD symptom and comorbid disorders including } \\
\text { oppositional defiant and conduct problems, executive functions, } \\
\text { learning problems, peer relations and defiance/aggression }\end{array}$ & S \\
\hline Executive function & BRIEF-Preschool ${ }^{80}$ & $\begin{array}{l}63 \text { items assessing executive functions within the home environment, } \\
\text { including working memory, mental set shifting, response inhibition, } \\
\text { emotional control and planning/organisation }\end{array}$ & Y \\
\hline
\end{tabular}

$\begin{array}{ll}\text { BRIEF }^{81} & 86 \text { items assessing executive functions in the home environment, } \\ & \text { including working memory, mental set shifting, response inhibition, } \\ & \text { emotional control, planning/organisation, organisation of materials, } \\ \text { initiation and behaviour monitoring }\end{array}$

Adaptive functioning ABAS-3: 0-5 years ${ }^{82} 241$ items assessing adaptive functioning skills, including Y communication, community use, preacademics, home living, health and safety, leisure, self-care, self-direction and social abilities

\begin{tabular}{|c|c|c|c|}
\hline & ABAS-3: 5-21 years ${ }^{82}$ & $\begin{array}{l}232 \text { items assessing adaptive functioning skills, including } \\
\text { communication, community use, functional academics, home living, } \\
\text { health and safety, leisure, self-care, self-direction and social abilities }\end{array}$ & $S$ \\
\hline Social skills & SSIS Rating Scale ${ }^{83}$ & $\begin{array}{l}79 \text { items assessing social skills, problem behaviours and academic } \\
\text { competence }\end{array}$ & $\mathrm{Y}, \mathrm{S}$ \\
\hline Sensory processing & Sensory Profile $2^{84}$ & $\begin{array}{l}86 \text { items assessing sensory processing, including auditory, visual, } \\
\text { taste/smell, movement, body position, touch, plus behavioural skills } \\
\text { including activity levels and emotional/social skills }\end{array}$ & $\mathrm{Y}, \mathrm{S}$ \\
\hline \multirow[t]{2}{*}{$\begin{array}{l}\text { Behavioural, } \\
\text { emotional, social } \\
\text { problems }\end{array}$} & CBCL: $1.5-5$ years $^{85}$ & $\begin{array}{l}100 \text { items assessing internalising and externalising problems, emotional } \\
\text { reactivity, anxiety/depression, somatic complaints, withdrawal, sleep } \\
\text { problems, attention problems and aggressive behaviours }\end{array}$ & Y \\
\hline & CBCL: 6-18 years ${ }^{86}$ & $\begin{array}{l}113 \text { items assessing internalising and externalising problems, emotional } \\
\text { reactivity, anxiety/depression, somatic complaints, withdrawal, sleep } \\
\text { problems, attention problems and aggressive behaviours }\end{array}$ & $S$ \\
\hline Pragmatic language & $\mathrm{CCC}-2^{87}$ & $\begin{array}{l}70 \text { items assessing speech, syntax, semantics, coherence, } \\
\text { inappropriate initiation, stereotyped language, use of context, non- } \\
\text { verbal communication, social relations and interests }\end{array}$ & $\mathrm{Y}, \mathrm{S}$ \\
\hline
\end{tabular}

ABAS-3, adaptive behaviour assessment system, 3rd edition; ADHD, attention deficit/hyperactivity disorder; BRIEF, behavior rating inventory of executive functions; CADS, Conners' attention deficit hyperactivity disorder scales; CBCL, child behavior checklist; CCC-2, children's communication checklist, 2nd edition; SRS-2, Social Responsiveness Scale, 2nd edition; SSIS, social skills improvement system. 
Diagnostic and Statistical Manual of Mental Disorders, Fifth Edition (DSM-5) ${ }^{39}$

If the results of the ADI-R and ADOS-2 are not in agreement regarding ASD classification, we will employ the research criteria proposed by Risi and colleagues to resolve ADI-R and ADOS-2 discordance. ${ }^{40}$ Use of these criteria relax the original ADI-R criteria that were developed to detect the formerly defined Autistic Disorder, ${ }^{38}$ to encompass the broader category of ASD outlined in the DSM-5. A research diagnosis of ASD is thus assigned to a participant if he/she meets criteria on the ADOS-2 (either autism or autism spectrum) and meets one of the following criteria on the ADI-R:

1. Meets ASD cut-off for the social reciprocity domain and either communication or restricted interests and repetitive behaviours domains.

2. Comes within one point for both social reciprocity and communication domains.

3. Meets ASD cut-off on one domain (either social reciprocity or communication) and comes within two points of the cut-off on the other domain (either social reciprocity or communication).

In addition to a research classification, a multidisciplinary expert panel will establish a clinical consensus diagnosis for all participants with NF1 who have completed the ASD assessment. This consensus diagnosis will be made according to DSM- 5 guidelines, using all available diagnostic information.

\section{Neuroimaging procedure \\ Mock training}

At the MCRI site, children will complete a 30 min training session in a mock MRI scanner which reproduces the physical environment of the real scanner including noise effects. This familiarises participants to the MRI environment, aims to lowers anxiety and provides practice at keeping still during the scanning session. ${ }^{41}$ Children who find the training sessions distressing and wish to withdraw from the neuroimaging component may do so at any time without affecting their ability to participate in the cognitive and behavioural assessment.

\section{MRI scan}

Neuroimaging data will be obtained on a 3-Tesla Siemens MAGNETOM Prisma MRI scanner with a 64-channel head coil at both MCRI and CHW sites. The magnetic resonance spectroscopy sequences, which will only be conducted at MCRI, will use a 32-channel head coil. The neuroimaging protocol comprises structural and functional sequences which will be completed in two $45 \mathrm{~min}$ sessions with a $30 \mathrm{~min}$ break in between (MCRI site) or in one 45 min session (CHW site). See table 3 for sequence details.

\section{Structural neuroimaging}

Three dimensional high-resolution structural T1-weighted magnetisation-prepared rapid gradient-echo (MPRAGE) images will be acquired to provide whole brain and regional grey and white matter (WM) volume, cortical thickness and other morphological features, as outlined in table 3. Children exhibiting high levels of movement during the MPRAGE sequence, will complete a second T1-weighted multiecho magnetisation-prepared rapid gradient-echo sequence, which uses navigator-based prospective motion correction to reduce artefact and improve structural image contrast, providing more accurate tissue segmentation. ${ }^{41-43}$

A T2-SPACE (Sampling Perfection with Application optimised Contrast with flip angle Evolution) protocol will be acquired to obtain T2-weighted anatomical images and provide information about the number and location of focal areas of WM hyperintensity that are common in NF1 (table 3) ${ }^{3344-46}$ The relationship between focal areas of high intensity and cognitive and behavioural deficits remains unclear. ${ }^{3347}$

\section{Multiband, multishell diffusion neuroimaging}

A multiband accelerated echo-planar imaging (EPI) sequences protocol, developed by the Centre for Magnetic Resonance Research (CMRR, University of Minnesota), will be acquired in order to obtain diffusion-weighted images (DWI) and examine brain microstructure through the identification of WM fibre tracts and their directionality. DWI measures the direction and extent of water diffusion through brain tissue, which is dependent on the underlying tissue structure, permitting examination of differences in cellular structure. ${ }^{48}{ }^{49}$ Diffusion parameters indicate changes in axonal properties. ${ }^{48} 49$ The multiband accelerated EPI protocol uses multiple shell acquisition to accelerate DWI volume coverage, and involves anterior-posterior phase encoding direction as well as standard and reverse phase encoded blipped image acquisition to correct for magnetic susceptibility-induced distortions related to the EPI acquisitions. ${ }^{4150} 51$ Three diffusion weighted shells will be acquired (table 3 ) to perform tractography and estimate WM microstructure, including traditional tensor metrics (fractional anisotropy, and mean, radial and axial diffusivity), as well as more advanced techniques that provide greater specificity to the microstructural properties, such as fibre density.

\section{Multiband resting state functional neuroimaging}

Resting state functional MRI (rs-fMRI) will be used to measure intrinsic functional connectivity between brain regions while subjects are at rest. During the sequence, participants are instructed to look at a white fixation cross on a black screen (table 3). Resting state connectivity is useful for studying abnormal neural network connectivity in NF1, and its relationship with cognitive and behavioural deficits. ${ }^{52}$

\section{Magnetic resonance spectroscopy}

Magnetic resonance spectroscopy (MRS) is an in vivo tool capable of non-invasively measuring brain metabolites. Only the MCRI site will acquire two GABA-edited magnetic resonance spectra using the localised 


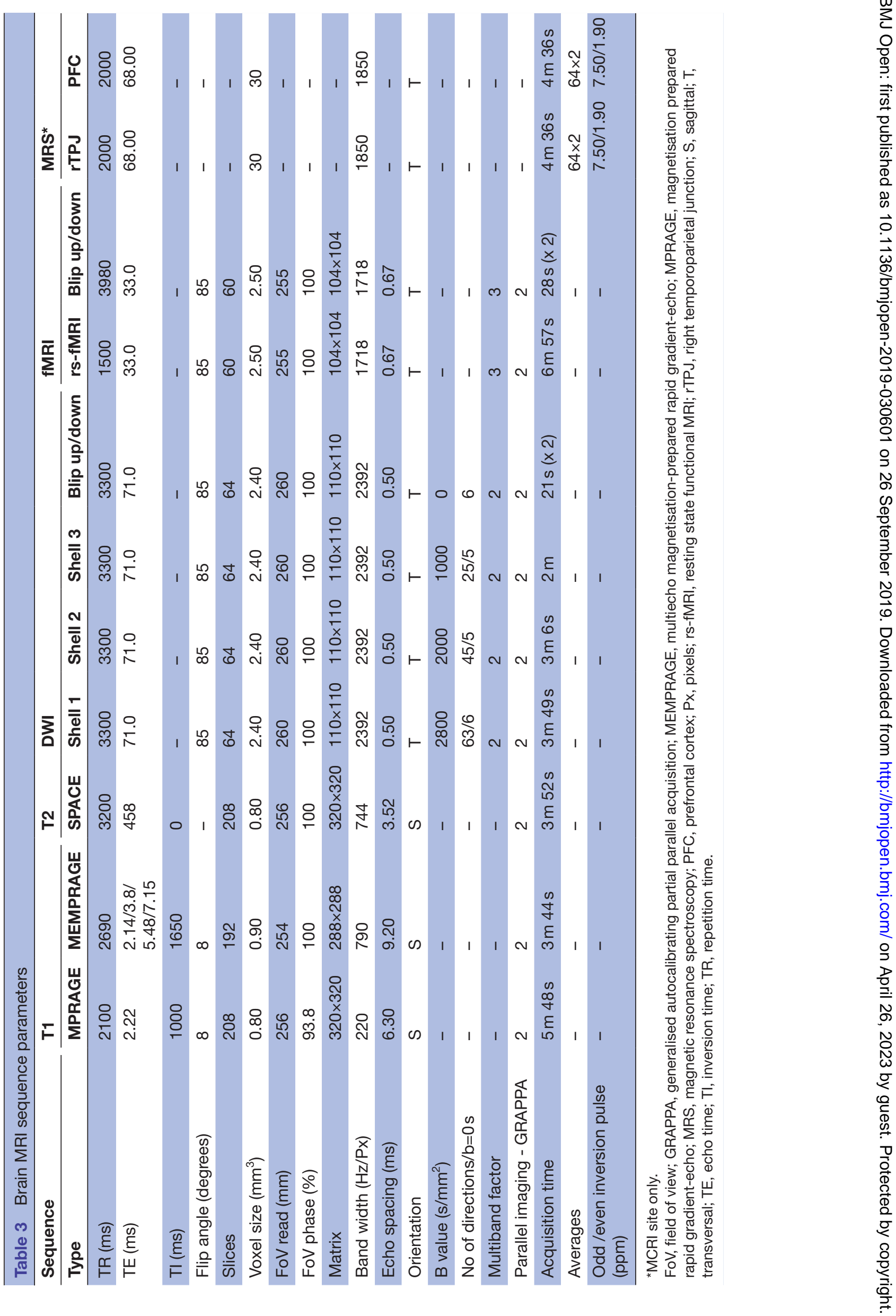


spectroscopy sequence MEGA-PRESS, developed by CMRR, to evaluate the animal model-derived hypothesis that alterations in GABA and glutamate systems underlie cognitive and social impairments in NF1. ${ }^{53-55}$ MEGAPRESS allows separation of GABA signals from stronger overlying signals of other metabolites. ${ }^{56}$ Voxels will be positioned in regions with consistency of field homogeneity within the prefrontal cortex (PFC) and the right temporoparietal junction (rTPJ), both of which are integral regions within the social brain network. ${ }^{57} 58$ The PFC voxel will be placed across the midline of the precentral sulcus, crossing across both hemispheres, in the medial PFC. The rTPJ voxel will be placed towards the rear border of the temporal and parietal lobes, in the posterior cerebral cortex. T1-weighted images will be used to guide MRS voxel placement.

\section{Data analysis}

Descriptive statistics will be used to establish the frequency of ASD in children with NF1. Between-group differences on general and social cognitive measures will be examined using analysis of variance, controlling for type 1 error. If particular demographic variables differ between groups (eg, age, sex, socioeconomic status) and are related to the outcome of interest, they will be introduced as a covariate using analysis of covariance (ANCOVA).

Within-group analysis for NF1 participants will identify the neuropsychological processes contributing to ASD symptoms and poor social functioning using (linear or logistic as appropriate) regressions within each age cohort. Composite variables will be created for variables that have high collinearity within the same domain. Regression models will be conducted separately for ASD symptomatology and social functioning as dependent variables. Only explanatory variables that significantly correlate with the dependent variables will be entered into each regression model, with a maximum of five predictor variables per model. Variables with the strongest correlation will be selected for the regression model.

Correlation and regression analyses will be used to identify associations between structural and functional brain markers, with cognitive, behavioural and ASD outcomes. To address the secondary aim, which is to compare the neuropsychological profiles as well as brain structure and function of children with NF1 and comorbid ASD to idiopathic ASD, statistical analyses examining group differences (ANCOVA and independent t-tests) between the idiopathic ASD and a subgroup of participants with NF1 and comorbid ASD will be conducted. Idiopathic ASD and NF1 cases will be matched on age, sex and intelligence.

\section{Sample size}

Cognitive and behavioural outcomes

We anticipate NF1 versus TD control between-group effect sizes to range from 0.65 to 1.0 (Cohen's d) based on estimates of general and social cognitive outcomes in previous studies. ${ }^{911} 15$ In order to detect a $d=0.65$ difference between the NF1 and TD control groups on continuous outcomes, with a minimum of $85 \%$ power and a significance level of 0.05 , we need to recruit at least 35 children per group in each age cohort (eg, younger children aged 3-5 years, and a school-aged cohort aged $6-15$ years). Within-group analyses performed within the NF1 group will require a larger sample to attain adequate power. For a multiple regression, with five independent variables in the model, accounting for an effect size $\left(\mathrm{f}^{2}\right)$ of 0.2 , power will be sufficiently high $(\beta=0.8)$ with a sample size of 70 . We thus require a minimum of 70 participants with NF1 in each age cohort. However, in order to attain a large enough NF1 with comorbid ASD subgroup $(n=35)$ for comparisons with TD control and idiopathic ASD groups, we estimate $\sim 200$ children with NF1 will need to be enrolled in the study. This assumes that $17 \%-18 \%$ of children with NF1 screened as part of the study will be diagnosed with comorbid ASD, which is consistent with previous estimations. ${ }^{17-19}$ If the target of 35 is not met, then we will endeavour to recruit extra NF1 participants until a subgroup size of 35 is achieved.

\section{Neuroimaging outcomes}

Published data demonstrate large effect sizes when comparing DWI and fMRI measures in individuals with NF1 to TD controls. ${ }^{59-61}$ Between-group independent $\mathrm{t}$-tests will be adequately powered $(\beta=0.80)$ to detect medium-to-large effect sizes (Cohen's $d=0.68$ ) with a minimum sample of $n=35$ per group. For the correlational analyses of DWI, rs-fMRI and behavioural data within the NF1 sample, power will be sufficiently high $(\beta=0.8)$ to detect moderate association $(r \geq 0.4)$ with a NF1 sample of $45{ }^{62}$

\section{Secondary outcomes}

We will recruit 35 idiopathic ASD participants. Sample size is based on (1) ANCOVA power analyses described above which indicate a sample size of 35 per group is sufficient to determine group differences on social cognitive measures; and (2) a previously published neuroimaging study involving 10 idiopathic ASD and 22 control participants which reported significant group differences in brain structure using structural MRI techniques. ${ }^{62}$

\section{Patient and public involvement}

Neither patient nor the public were involved in the development of the research questions, selection of outcome measures, study design or study conduct.

\section{ETHICS AND DISSEMINATION}

Any protocol modifications will be communicated to the study team and ethics committees. This study will be conducted in compliance with this protocol, the conditions of the ethics committee approval, the NHMRC National Statement on ethical Conduct in Human Research (2007), and the Note for Guidance on Good Clinical Practice (CPMP/ICH-135/95). Written informed consent will be obtained from all participants. During the informed consent process, a member of the research 
team will provide information about the study including the study objectives, potential risks and benefits, inconveniences and the participants' rights and responsibilities. Questions about the study will be addressed in detail. As participants are minors, written informed consent will be obtained from their parent/legal guardian.

The findings of this study will be presented at conferences and published in peer-reviewed journals. Only aggregated data will be reported in publications and presentations with individual identifying information removed. The investigator team will write all articles submitted for peer-reviewed publications and authorship inclusion and order will be guided by levels of contribution.

\section{DISCUSSION}

ASD is a highly complex polygenic disorder in which children experience significant impairments in social interaction, communication and restricted interests and repetitive behaviours. However, the aetiology of these impairments remains poorly understood, which limits insights into neurobiological mechanisms and in turn, targeted pharmacological treatment. Studying ASD in children with NF1 offers a complementary approach to studying the idiopathic population by allowing us to systematically explore whether there are distinct neurobiological, cognitive and behavioural ASD phenotypes related to mutations at NF1. There will be a number of important novel outcomes from this study. First, it will combine gold standard diagnostic assessments with extensive cognitive and behavioural phenotyping to estimate the frequency of ASD and characterise the problem behaviours in children with NF1 as young as 3 years of age. Second, this study will characterise the social phenotype of NF1 and model the interrelationships between various levels of social functioning (eg, social interactions, information processing and adjustment), and how abnormal functioning is associated with ASD symptomatology. Third, this study seeks to map brain structure and function onto a comprehensive set of cognitive, behavioural and functional outcomes, encompassing general and social cognition, ASD symptom profiles, academic achievement and adaptive functioning. By identifying the neurobiological and cognitive factors influencing functional outcomes, there is potential to provide insight into whether the genetic homogeneity in NF1 results in a unique, more consistent behavioural and cognitive phenotype than that seen in the idiopathic ASD population. Characterisation of the cognitive, behavioural and neurobiological phenotype in NF1-related ASD may assist in determining novel targets for future intervention studies aimed at improving social outcomes in ASD, as well as clinical populations with social difficulties more broadly, to improve patient outcomes. Finally, identifying neural correlates of social dysfunction and ASD in NF1 may provide researchers with valid surrogate endpoints for clinical trials, which would be particularly useful in proof of concept pilot studies and to assist optimising aspects of trial design such as dose refinement.

\section{Author affiliations}

${ }^{1}$ Brain and Mind, Murdoch Children's Research Institute, Parkville, VIC, Australia ${ }^{2}$ Department of Paediatrics, Faculty of Medicine, Dentistry and Health Science, University of Melbourne, Parkville, VIC, Australia

${ }^{3}$ Kids Neuroscience Centre, The Children's Hospital at Westmead, Westmead, NSW, Australia

${ }^{4}$ Discipline of Child and Adolescent Health, University of Sydney Medical School, Westmead, NSW, Australia

${ }^{5}$ Center for Neuroscience and Behavioral Medicine, Children's National Health System, Washington, DC, United States

${ }^{6}$ Departments of Pediatrics and Psychiatry, The George Washington University School of Medicine, Washington, DC, United States

${ }^{7}$ Children's Hospital Education Research Institute, The Children's Hospital at Westmead, Westmead, NSW, Australia

${ }^{8}$ School of Psychology, Deakin University, Burwood, VIC, Australia

${ }^{9}$ Brain Dynamics Centre, Westmead Institute for Medical Research, University of Sydney, Westmead, NSW, Australia

${ }^{10}$ Developmental Imaging, Murdoch Children's Research Institute, Parkville, VIC, Australia

${ }^{11}$ Department of Paediatrics, Monash University, Clayton, VIC, Australia

${ }^{12}$ Department of Neurology, Royal Children's Hospital Melbourne, Parkville, VIC, Australia

${ }^{13}$ Neuroscience Research Australia, University of New South Wales, Randwick, NSW, Australia

${ }^{14}$ Imaging Department, Royal Children's Hospital Melbourne, Parkville, VIC, Australia

${ }^{15}$ Murdoch Children's Research Institute, Parkville, VIC, Australia

Contributors JMP, NAP, KSW, BB and KNN developed the original concept of the study, wrote the grant applications and drafted the original protocol and methodology. KMH, AC, MR, AM, VA, TS, KW, MSK, MS, FL, JL, CDR, GD and MK provided additional advice on the study design, analysis techniques and/or statistical methods. JMP, KMH and NAP drafted this protocol paper. All authors commented on the final preparation of the protocol and have read and approved the final manuscript.

Funding This work was supported by a US Department of Defense Investigator Initiated Research Award and an Exploration-Hypothesis Development Award as part of the Neurofibromatosis Research Program, respective grant numbers W81XWH-15-1-0619 and W81XWH-16-1-0408; a Murdoch Children's Research Institute Clinician Scientist Fellowship awarded to JMP; a NHMRC Postgraduate Scholarship awarded to AC; and the Children's Tumour Foundation in Australia.

Competing interests None declared.

Patient consent for publication Not required.

Ethics approval This study has been granted approval by the Human Research Ethics Committee of the Royal Children's Hospital (HREC35118), Sydney Children's Hospitals Network and the Institutional Review Board of the Children's National Health System.

Provenance and peer review Not commissioned; externally peer reviewed.

Open access This is an open access article distributed in accordance with the Creative Commons Attribution Non Commercial (CC BY-NC 4.0) license, which permits others to distribute, remix, adapt, build upon this work non-commercially, and license their derivative works on different terms, provided the original work is properly cited, appropriate credit is given, any changes made indicated, and the use is non-commercial. See: http://creativecommons.org/licenses/by-nc/4.0/.

\section{REFERENCES}

1. Tordjman S, Somogyi E, Coulon N, et al. Gene $\times$ Environment interactions in autism spectrum disorders: role of epigenetic mechanisms. Front Psychiatry 2014;5.

2. Sandin S, Lichtenstein P, Kuja-Halkola R, et al. The heritability of autism spectrum disorder. JAMA 2017;318:1182-4.

3. Tick B, Bolton P, Happé F, et al. Heritability of autism spectrum disorders: a meta-analysis of twin studies. J Child Psychol Psychiatry 2016;57:585-95. 
4. Anney R, Klei L, Pinto D, et al. Individual common variants exert weak effects on the risk for autism spectrum disorders. Hum $\mathrm{Mol}$ Genet 2012;21:4781-92

5. Buxbaum JD, Daly MJ, Devlin B, et al. The autism sequencing Consortium: large-scale, high-throughput sequencing in autism spectrum disorders. Neuron 2012;76:1052-6.

6. Pinto D, Delaby E, Merico D, et al. Convergence of genes and cellular pathways dysregulated in autism spectrum disorders. Am J Hum Genet 2014;94:677-94.

7. Winden KD, Ebrahimi-Fakhari D, Sahin M. Abnormal mTOR activation in autism. Annu Rev Neurosci 2018;41:1-23.

8. Evans DG, Howard E, Giblin C, et al. Birth incidence and prevalence of tumor-prone syndromes: estimates from a UK family genetic register service. Am J Med Genet A 2010;152A:327-32.

9. Hyman SL, Shores A, North KN. The nature and frequency of cognitive deficits in children with neurofibromatosis type 1. Neurology 2005;65:1037-44.

10. Lehtonen A, Howie E, Trump D, et al. Behaviour in children with neurofibromatosis type 1: cognition, executive function, attention, emotion, and social competence. Dev Med Child Neurol 2013;55:111-25.

11. Chisholm AK, Anderson VA, Pride NA, et al. Social function and autism spectrum disorder in children and adults with neurofibromatosis type 1: a systematic review and meta-analysis Neuropsychol Rev 2018;28:317-40.

12. Lewis AK, Porter MA, Williams TA, et al. Facial emotion recognition, face scan paths, and face perception in children with neurofibromatosis type 1. Neuropsychology 2017;31:361-70.

13. Allen T, Willard VW, Anderson LM, et al. Social functioning and facial expression recognition in children with neurofibromatosis type 1. J Intellect Disabil Res 2016;60:282-93.

14. Huijbregts S, Jahja R, De Sonneville L, et al. Social information processing in children and adolescents with neurofibromatosis type 1. Dev Med Child Neurol 2010;52:620-5.

15. Payne JM, Porter M, Pride NA, et al. Theory of mind in children with neurofibromatosis type 1. Neuropsychology 2016;30:439-48.

16. Morris SM, Acosta MT, Garg S, et al. Disease burden and symptom structure of autism in neurofibromatosis type 1: a study of the International NF1-ASD Consortium team (INFACT). JAMA Psychiatry 2016;73:1276-84.

17. Plasschaert E, Descheemaeker M-J, Van Eylen L, et al. Prevalence of autism spectrum disorder symptoms in children with neurofibromatosis type 1. Am J Med Genet B Neuropsychiatr Genet 2015;168B:72-80.

18. Eijk S, Mous SE, Dieleman GC, et al. Autism spectrum disorder in an unselected cohort of children with neurofibromatosis type 1 (NF1). $J$ Autism Dev Disord 2018;48:2278-85.

19. Garg S, Green J, Leadbitter K, et al. Neurofibromatosis type 1 and autism spectrum disorder. Pediatrics 2013;132:e1642-8.

20. Lai M-C, Lombardo MV, Baron-Cohen S. Autism. Lancet 2014;383:896-910.

21. Noll RB, Reiter-Purtill J, Moore BD, et al. Social, emotional, and behavioral functioning of children with NF1. Am J Med Genet $A$ 2007;143A:2261-73

22. Payne JM, Hyman SL, Shores EA, et al. Assessment of executive function and attention in children with neurofibromatosis type 1: relationships between cognitive measures and real-world behavior. Child Neuropsychol 2011;17:313-29.

23. Wang DL, Smith KB, Esparza S, et al. Emotional functioning of patients with neurofibromatosis tumor suppressor syndrome. Genet Med 2012;14:977-82

24. Beauchamp MH, Anderson V. SOCIAL: an integrative framework for the development of social skills. Psychol Bull 2010;136:39-64.

25. Craig F, Margari F, Legrottaglie AR, et al. A review of executive function deficits in autism spectrum disorder and attention-deficit/ hyperactivity disorder. Neuropsychiatr Dis Treat 2016;12:1191-202.

26. Garg S, Plasschaert E, Descheemaeker M-J, et al. Autism spectrum disorder profile in neurofibromatosis type I. J Autism Dev Disord 2015;45:1649-57.

27. Christensen DL, Braun KVN, Baio J, et al. Prevalence and Characteristics of Autism Spectrum Disorder Among Children Aged 8 Years - Autism and Developmental Disabilities Monitoring Network 11 Sites, United States, 2012. MMWR Surveill Summ 2018;65:1-23.

28. Garg S, Heuvelman H, Huson S, et al. Sex bias in autism spectrum disorder in neurofibromatosis type 1. J Neurodev Disord 2016;8:26.

29. Bent CA, Dissanayake C, Barbaro J. Mapping the diagnosis of autism spectrum disorders in children aged under 7 years in Australia, 2010-2012. Med J Aust 2015;202:317-20.

30. Klein-Tasman BP, Janke KM, Luo W, et al. Cognitive and psychosocial phenotype of young children with neurofibromatosis-1. J Int Neuropsychol Soc 2014;20:88-98.
31. Pennington BF, Welsh M. Neuropsychology and developmental psychopathology. J Child Psychol Psychiatry 1995;37:51-87.

32. Wang $Y$, Kim E, Wang X, et al. Erk inhibition rescues defects in fate specification of $\mathrm{Nf} 1$-deficient neural progenitors and brain abnormalities. Cell 2012;150:816-30.

33. Payne JM, Moharir MD, Webster R, et al. Brain structure and function in neurofibromatosis type 1: current concepts and future directions. $J$ Neurol Neurosurg Psychiatry 2010;81:304-9.

34. Violante IR, Patricio M, Bernardino I, et al. GABA deficiency in NF1: a multimodal [11C]-flumazenil and spectroscopy study. Neurology 2016;87:897-904.

35. Cui Y, Costa RM, Murphy GG, et al. Neurofibromin regulation of ERK signaling modulates GABA release and learning. Cell 2008;135:549-60.

36. Neurofibromatosis. Conference statement. National Institutes of health consensus development conference. Arch Neurol 1988;45:575-8.

37. Payne JM, North KN. Neurofibromatosis type 1. In: Goldstein $\mathrm{S}$, Reynolds CR, eds. Handbook of neurodevelopmental and genetic disorders in children. 2nd edn. New York: Guilford Press, 2010: 322-37.

38. Rutter M, Le Couteur A, Lord C. ADI-R: autism diagnostic InterviewRevised (ADI-R). Los Angeles, CA: Western Psychological Services, 2003.

39. Lord C, Rutter M, DiLavore PC, et al. Autism diagnostic observation schedule, second edition: ADOS-2. Torrance: Western Psychological Services, 2012

40. Risi S, Lord C, Gotham K, et al. Combining information from multiple sources in the diagnosis of autism spectrum disorders. J Am Acad Child Adolesc Psychiatry 2006;45:1094-103.

41. Silk TJ, Vilgis V, Adamson $\mathrm{C}$, et al. Abnormal asymmetry in frontostriatal white matter in children with attention deficit hyperactivity disorder. Brain Imaging Behav 2016;10:1080-9.

42. Tisdall MD, Hess AT, Reuter M, et al. Volumetric navigators for prospective motion correction and selective reacquisition in neuroanatomical MRI. Magn Reson Med 2012;68:389-99.

43. van der Kouwe AJW, Benner T, Salat DH, et al. Brain morphometry with multiecho MPRAGE. Neuroimage 2008;40:559-69.

44. Van Es S, North KN, McHugh K, et al. Mri findings in children with neurofibromatosis type 1: a prospective study. Pediatr Radiol 1996;26:478-87.

45. Sevick RJ, Barkovich AJ, Edwards MS, et al. Evolution of white matter lesions in neurofibromatosis type 1: Mr findings. AJR Am J Roentgenol 1992;159:171-5.

46. Lopes Ferraz Filho JR, Munis MP, Soares Souza A, et al. Unidentified bright objects on brain MRI in children as a diagnostic criterion for neurofibromatosis type 1. Pediatr Radiol 2008;38:305-10.

47. DiPaolo DP, Zimmerman RA, Rorke LB, et al. Neurofibromatosis type 1: pathologic substrate of high-signal-intensity foci in the brain. Radiology 1995;195:721-4.

48. Hagmann $\mathrm{P}$, Jonasson L, Maeder $\mathrm{P}$, et al. Understanding diffusion MR imaging techniques: from scalar diffusion-weighted imaging to diffusion tensor imaging and beyond. Radiographics 2006;26(Suppl 1):S205-S223.

49. Le Bihan D, Mangin JF, Poupon C, et al. Diffusion tensor imaging concepts and applications. J Magn Reson Imaging 2001;13:534-46.

50. Auerbach EJ, Xu J, Yacoub E, et al. Multiband accelerated spin-echo echo planar imaging with reduced peak rf power using time-shifted rf pulses. Magn Reson Med 2013;69:1261-7.

51. Xu J, Moeller S, Auerbach EJ, et al. Evaluation of slice accelerations using multiband echo planar imaging at 3 T. Neuroimage 2013;83:991-1001.

52. Tomson SN, Schreiner MJ, Narayan M, et al. Resting state functional MRI reveals abnormal network connectivity in neurofibromatosis 1 . Hum Brain Mapp 2015;36:4566-81.

53. Shilyansky C, Lee YS, Silva AJ. Molecular and cellular mechanisms of learning disabilities: a focus on NF1. Annu Rev Neurosci 2010;33:221-43.

54. Gonçalves J, Violante IR, Sereno J, et al. Testing the excitation/ inhibition imbalance hypothesis in a mouse model of the autism spectrum disorder: in vivo neurospectroscopy and molecular evidence for regional phenotypes. Mol Autism 2017;8:47.

55. Molosh Al, Johnson PL, Spence JP, et al. Social learning and amygdala disruptions in NF1 mice are rescued by blocking p21activated kinase. Nat Neurosci 2014:17:1583-90.

56. Mullins PG, McGonigle DJ, O'Gorman RL, et al. Current practice in the use of MEGA-PRESS spectroscopy for the detection of GABA Neuroimage 2014;86:43-52.

57. Schurz M, Radua J, Aichhorn M, et al. Fractionating theory of mind: a meta-analysis of functional brain imaging studies. Neurosci Biobehav Rev 2014:42:9-34. 
58. Bzdok D, Schilbach L, Vogeley K, et al. Parsing the neural correlates of moral cognition: ale meta-analysis on morality, theory of mind, and empathy. Brain Struct Funct 2012;217:783-96.

59. Zamboni SL, Loenneker T, Boltshauser E, et al. Contribution of diffusion tensor MR imaging in detecting cerebral microstructura changes in adults with neurofibromatosis type 1. AJNR Am J Neuroradiol 2007;28:773-6.

60. Shilyansky C, Karlsgodt KH, Cummings DM, et al. Neurofibromin regulates corticostriatal inhibitory networks during working memory performance. Proc Natl Acad Sci U S A 2010;107:13141-6.

61. van Engelen SJPM, Krab LC, Moll HA, et al. Quantitative differentiation between healthy and disordered brain matter in patients with neurofibromatosis type I using diffusion tensor imaging AJNR Am J Neuroradiol 2008;29:816-22.

62. Faul F, Erdfelder E, Buchner A, et al. Statistical power analyses using $\mathrm{G}^{*}$ Power 3.1: tests for correlation and regression analyses. Behav Res Methods 2009;41:1149-60.

63. Wechsler D. Wechsler preschool and primary scale of intelligence. 4th edn. San Antonio, TX: Pearson, 2012.

64. Wechsler D. Wechsler intelligence scale for children. 5th edn. Pearson Assessment, 2016.

65. Manly $\mathrm{T}$, Robertson IH, Anderson V, et al. The test of everyday attention for children. London: Battley Brothers, 1999.

66. Espy KA. The shape school: assessing executive function in preschool children. Dev Neuropsychol 1997;13:495-9.

67. Korkman M, Kirk U, Kemp S. The NEPSY second edition (NEPSY II) scoring manual. Bloomington, MN: : Pearson, 2007.

68. Wechsler D. Wechsler intelligence scales for children. 5th edn. San Antonio: The Psychological Corporation, 2016.

69. Welsh MC, Pennington BF, Groisser DB. A normative-developmental study of executive function: a window on prefrontal function in children. Dev Neuropsychol 1991;7:131-49.

70. Wechsler D. Wechsler individual achievement test. 2 nd edn. New York: Psychological Corporation, 2002.

71. Benton AL, Sivan AB, Hamsher K, et al. Contributions for neuropsychological assessment. 2nd edn. Orlando, FL: Psychological Assessment Resources, 1994

72. Baron-Cohen S, Wheelwright S, Spong A, et al. Are intuitive physics and intuitive psychology independent? A test with children with Asperger syndrome. J Dev Learn Disord 2000;5:47-78.
73. Baron-Cohen S, O'Riordan M, Stone V, et al. A new test of social sensitivity: detection of faux PAS in normal children and children with Asperger syndrome. J Autism Dev Disord 1999;29:407-18.

74. Happé FG. An advanced test of theory of mind: understanding of story characters' thoughts and feelings by able autistic, mentally handicapped, and normal children and adults. J Autism Dev Disord 1994;24:129-54.

75. Wiig EH, Secord W, Semel E. Clinical Evaluation of Language Fundamentals - Preschool, 2nd Edition, Australian and New Zealand Standardised Edition (CELF P-2 Australian and New Zealand). Marrickville NSW Australia: Harcourt Assessment, 2006.

76. Semel E, Wiig EH, Secord WA. Clinical evaluation of language fundamentals. 4th edn. Sydney: Harcourt Assessment, 2003.

77. Constantino JN, Gruber CP. Social responsiveness scale. 2nd edn. Los Angeles: Western Psychological Services, 2012.

78. Conners CK. Conners' rating Scales-revised. New York: Multi-Health Systems Inc, 1997.

79. Conners CK. Conners. 3rd edn. Toronto: Multi-Health Systems Inc, 2009.

80. Gioia GA, Espy KA, Isquith PK. Behavior rating inventory of executive function, preschool version. Lutz, FL: Psychological Assessment Resources, Inc, 2003.

81. Gioia GA, Isquith PK, Guy SC, et al. Behavior rating inventory of executive function: professional manual. Odessa: PAR, Inc, 2000.

82. Harrison P, Oakland T. Adaptive behavior assessment system. 3rd edn, 2015.

83. Gresham FM, Elliott SN. Social skills improvement system rating scales. Minneapolis: NCS Pearson, 2008.

84. Dunn W. Sensory Profile-2. UK: Pearson, 2014.

85. Achenbach TM, Rescorla LA. Manual for the ASEBA preschool forms and profiles. Burlington, VT: University of Vermont Department of Psychiatry, 2000.

86. Achenbach TM, Rescorla LA. Manual for the ASEBA school-age forms and profiles. Burlington, VT: University of Vermont, Research Center for Children, Youth, and Families, 2001.

87. Bishop DVM. Children's communication Checklist-2. San Antonio, TX: Pearson, 2006 